\title{
MUC1 and carbohydrate associated antigens in head and neck squa- mous cell carcinoma: our experience
}

\author{
María Virginia Croce*, Martín E. Rabassa, Amada Segal-Eiras
}

Centre of Basic and Applied Immunological Research (CINIBA), Faculty of Medical Sciences, National University of La Plata, Argentina.

Article Info

\section{Article Notes}

Received: May 06, 2018

Accepted: May 30, 2018

\section{${ }^{*}$ Correspondence:}

Dr. María Virginia Croce, Centre of Basic and Applied Immunological Research (CINIBA), Faculty of Medical

Sciences, National University of La Plata, Argentina;

Email: crocevir@hotmail.com

(C) 2018 Croce MV. This article is distributed under the terms of the Creative Commons Attribution 4.0 International License.
Head and neck cancer constitutes the sixth cause of cancer worldwide with an age-standardized rate of 9.1 cases per 100,000 inhabitants, not including thyroid tumors ${ }^{1}$. Most stage I/II patients have a very good prognosis while those with higher stages of the disease show a poor overall survival being the presence of lymph node metastases the main factor ${ }^{2}$. Development of tumor markers either prognostic as well as to detect early recurrences or therapypredictive would have critical importance. In the last years, several publications related to immunotherapy $y^{3,4}$, genomics ${ }^{5-7}$ and viral carcinogenesis $8^{-10}$ have explored new biological targets and markers for this disease.

The alteration in MUC1 and carbohydrate associated antigens would play a role in head and neck squamous cell carcinoma (HNSCC) development/dissemination and, in consequence, may bring relevant information to clinical aspects. MUC1 is expressed by normal and neoplastic benign and malignant epithelial cells; it is translated as a single polypeptide that undergoes autocleavage into two subunits. The MUC1 N-terminal subunit (MUC1-N) contains highly conserved tandem repeats of 20 amino acids that are extensively modified by $O$-linked glycans, forms a stable noncovalent complex with the C-terminal transmembrane subunit (MUC1-C) and is thereby anchored to the cell surface. In carcinomas, MUC1 is often highly overexpressed losing its apical polarization, displays altered mRNA splicing variants and aberrant glycosylation ${ }^{11}$. 13. Tumor MUC1-N exhibits the Core 10 -glycans and, additionally, is highly sialylated, which causes premature termination of chain elongation and formation of truncated sugar branches with the expression of different terminal glycan epitopes ${ }^{14}$. In Figure 1, the Mucin type 0-glycosylation biosynthetic pathway is summarized; although the antigens showed have been found both in normal and neoplastic squamous epithelia, some have been mainly detected in malignant tumors such as Tn hapten (Tn), sialylTn (sTn), and sialyl Lewis $\mathrm{x}$ (sLex).

O-glycosylation is initiated by one of the several ppGalNAc transferases and, if no further glycans are added, results in the expression of the Tn antigen. In tumors, short glycans might be generated by early sialylation (sTn), while in normal keratinized or non-keratinized stratified epithelium, core structures are developed by core synthases (C1GALT1 and GCNTs). Core synthesis and polylactose chain extension results in $\mathrm{T}$ antigen and Lac (Lactose) or sLac (sialyl Lactose) expression, respectively. Sialylation and fucosylation of the outermost part might give rise to antigens of the 


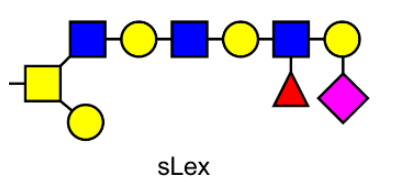

sLex


Lex/y

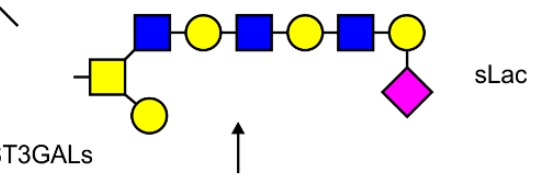

FUTs



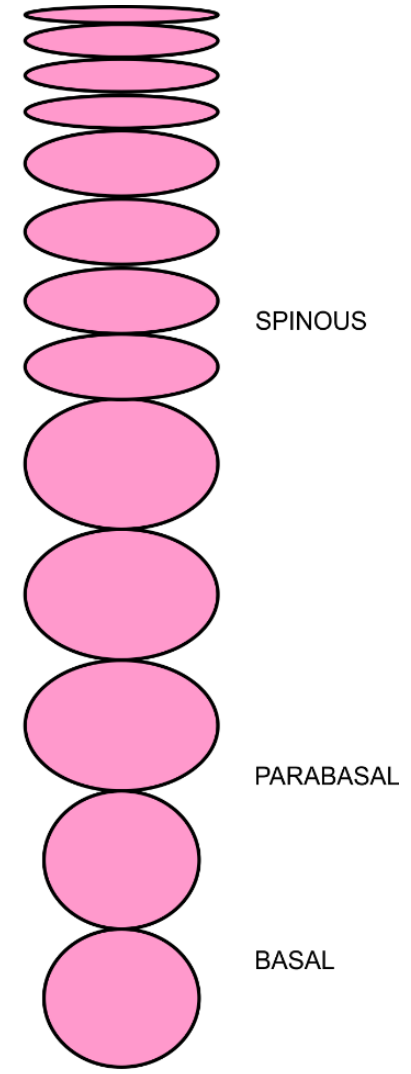

EPITHELIAL LAYER

Figure 1: Mucin type O-glycosylation biosynthetic pathway.

Lewis group (in type 1 lactose chains Lea or $b$ and in type 2 chains Ley or $\mathrm{x}$ ). Finally, some tumors are capable of sLex synthesis, a glycan structure related to cell adhesion and metastatic dissemination.

In an early study, we determined the expression of MUC1-N, Tn, Lewis x (Lex), sLex and Lewis y (Lex) in specimens of advanced carcinoma of the larynx by immunohistochemistry (IHC); additionally, an extensive procedure favoring the detection and isolation of MUC1 from tumor samples was performed ${ }^{15}$. Taking into account that the antigens evaluated have been reported to be factors associated with increased metastatic potential ${ }^{16,17}$, we established a new larynx carcinoma cell line which would provide the basis for further experiments ${ }^{18}$. The tumor belonged to a patient with a poorly differentiated carcinoma with lymphatic node metastasis; an accurate antigenic characterization showed the expression of MUC1-N, Tn, Lex, sLex and Ley antigens. The similar immunohistochemical (IHC) pattern of expression of MUC1 and sLex as well as the fact that subcellular fractions showed the same molecular weight (MW) bands by Western-Blot (WB) suggested that MUC1 may be its carrier. An expanded study on the above panel of antigens as tumor markers in HNSCC was reported ${ }^{19}$. Specimens from patients with tongue, larynx, oral cavity, maxillary sinus, tonsillar ring and pharynx tumors were included. The study also included normal tissue samples from the same locations, obtained from patients without any history of HNSCC or local inflammation. Tumor samples showed a high expression of the panel of antigens while no correlation with tumor localization was detected; the similar IHC pattern of reactivity of MUC1-N and sLex and also Ley was a remarkable finding. Furthermore, WB analysis from isolated subcellular fractions showed similar MW bands with anti-MUC1 and sLex confirming previous results; Tn showed the same bands. In this report, we compared the IHC expression of 6 tumor samples with their matched regional metastatic lymphatic nodes; in three cases, metastatic cells presented a coincident expression of MUC1-N; also, sLex, Lex, and Tn were detected.

An IHC approach was developed to examine the effect of desialylation on peptidic as well as carbohydrate MUC1 
(Tn, T, sialyl Tn, sialyl T) epitope reactivity ${ }^{20}$. In a group of 24 HNSSC samples (pharynx, larynx, maxillary sinus, and oral cavity), we demonstrated that the treatment of samples with neuraminidase resulted increasing MUC1 and Tn expression while a negative reaction was found in the only one sTn+ tumor after treatment. Furthermore, Tn epitope was not detected without desialylation, while neuraminidase treatment allowed 10 samples to become positive. Again, WB analysis showed the same MW bands for MUC1 and Tn.

In 53 HNSCC patients, anti-MUC1 humoral immune response was studied; from each patient, a tumor specimen as well as a pretreatment serum sample were obtained $^{21}$. Comparison between MUC1-N and MUC1-C subunit expression in tumor samples was performed. Taking into account that release of MUC1-N from the cell surface occurs and it may be detected in serum samples, MUC1-N serum levels were measured. We also searched for a possible humoral anti-MUC1-N immune response by detection of free IgG/IgM anti-MUC1-N antibodies by ELISA and circulating immune complexes (CIC) by precipitation in polyethylene glycol 3.5\%. Finally, to probe that CIC antigen was MUC1, IgG-CIC was isolated by protein A-sepharose CL-4B affinity chromatography followed by SDS-PAGE and WB. It was found that MUC1-C was widely expressed in tumor samples; serum MUC1-N levels were higher in cancer patients than controls $(p=0.005)$; free IgG/IgM anti-MUC1 serum levels in HNSCC were lower than controls and only IgG antibodies positively correlated with MUC1-N levels, which was the first indication that an anti-MUC1-N immune response was mounted in these tumors. In HNSCC serum samples, high levels of CIC were detected statistically associated with tumor stage and differentiation. It was conclusively demonstrated that CIC antigenic moiety was MUC1-N.

To further demonstrate the differential expression of the panel of antigens selected and their possible performance as tumor markers, comparison with normal counterparts was performed. Respect to tumor samples, normal squamous epithelium of larynx, pharynx, tonsillar ring, maxillary sinus, oral cavity, and tongue showed a much lower expression of MUC1-N in the cytoplasm at several basal and superficial cells. Carbohydrate associated antigens showed a more extended expression than MUC1 although lower than malignant cells; staining was usually restricted to a few cells belonging to the superficial layer while, in some cases, basal and intermediate strata were reactive; the pattern of distribution was homogeneous, mostly a moderate cytoplasmic reaction. Lex showed the strongest reaction followed by Ley and finally, $\operatorname{Tn}^{19,} 22$. Again, among glycan antigens, Lex was the most reactive with a predominant linear pattern; sLex, Ley, Tn, and sTn were also reactive but with a more restricted and lower expression.
As it was pointed above, tumor MUC1 differs from that expressed in normal cells, both in its biochemical features and its cellular distribution as well. It has been demonstrated that these phenomena are related; on one hand, hypoglysation impacts the stability and subcellular localization of MUC1 while increased sialylation may result on premature termination of sugar branches with the generation of $\mathrm{Tn} / \mathrm{T}$ antigens or overexpression of sLex/sLea antigens. Hypoglycosylation without enhanced degradation may potentiate MUC1 oncogenic signaling by decreasing its cell surface levels and increasing intracellular accumulation ${ }^{13}$. The overexpression of MUC1 in different tumor locatizations has been linked to the activation of several pathways that are known to be of importance to cancer dissemination ${ }^{23-27}$. Furthermore, sLex/sLea are ligands of selectins and mediate tumor adhesion to vascular endothelia associated with a worse prognosis in several malignant neoplasms ${ }^{28}$. To unravel the relationship of MUC1 and the panel of glycan antigens with dissemination in HNSCC, we developed a study including primary tumors, metastatic lymphatic nodes and local recurrences by means of histological detection and subcellular isolation. The study included 125 patients with HNSCC (oral cavity, larynx, pharynx, nasal cavity, and paranasal sinuses) with different histopathological differentiation and at different stages of disease ${ }^{29}$. Our findings supported previous reports which indicated a high expression of MUC1 and related Lewis antigens in HNSCC; interestingly, MUC1-C was highly expressed and both MUC1 subunits were mainly found at cytoplasmic level while plasmatic fractions were not so frequently reactive, but nuclei fractions were also stained in several specimens; these findings sustained the involvement of MUC1 on dissemination. On the other hand, expression did not show differences among metastatic lymph nodes and primary tumors also considering analysis of paired samples, while tumor recurrences showed a lower expression than primary tumors, possibly because recurrences consisted on highly undifferentiated specimens. By an exhaustive methodology, we isolated MUC1 which was reactive with sLex and Ley antigens which was in agreement with our previous reports and suggested that MUC1 may be a carrier of these carbohydrates. Differentiation was the only variable that showed significant differences in relation to MUC1 expression; a notable feature was the variation of MUC1-C pattern of expression from linear in normal samples to cytoplasmic and nuclear in less differentiated primary tumors. Particularly, Lex expression was found to be inversely correlated with tumor T classification and associated with well-differentiated tumors. Considering this last finding, the prognostic utility of Lewis antigens in HNSCC was evaluated. With that purpose, we conducted a prospective research including histological samples from 79 patients with primary HNSCC. Lex and sLex expression 
were detected by immunohistochemistry; patient's data, progression free, and overall survival were documented ${ }^{30}$. By IHC, Lex was detected in $43 \%$ tumor samples, while sLex only in $14 \%$. Lex expression showed a positive correlation with tumor differentiation and a better overall survival for Lewis $\mathrm{x}+$ patients was detected; Cox's regression analysis showed that Lex is an independent predictor of better overall survival. A study on the enzymes implicated on patients' survival was performed; an in silico analysis was developed which supported the presence of deregulated fucosyl (FUT4) and sialyltransferase (ST3GAL4) in Lex synthetic pathway. These results suggested that Lex expression was associated with a better outcome in patients with HNSCC.

Considering the importance of HNSCC, substantial improvement has been generated in both biological as well as clinical aspects: 1-MUC1 and carbohydrate associated antigens are widely expressed in this tumor localization; 2- MUC1 may be a carrier of sLex and Ley; 3- MUC1 is immunogenic in HNSCC patients, and 4-Lex is a marker of good prognosis in these patients.

\section{Acknowledgments}

This study was supported by the National University of La Plata (Research grant number M153-M198).

Prof. Maria Virginia Croce and Dr. Martin E. Rabassa are members of the Research Career of the Comisión de Investigaciones Científicas of the provincia de Buenos Aires.

\section{Conflict of interest}

The authors declare that they have no financial relationship that may lead to a conflict of interest in relation to the submitted manuscript.

\section{References}

1. Ferlay J, Soerjomataram I, Ervik M, et al. Cancer Incidence and Mortality Worldwide: IARC CancerBase No. 11. Lyon, France: International Agency for Research on Cancer. 2013.

2. Lydiatt WM, Patel SG, O'Sullivan B, et al. Head and Neck cancers-major changes in the American Joint Committee on cancer eighth edition cancer staging manual. CA Cancer J Clin. 2017; 67(2): 122-137.

3. Shayan G, Kansy BA, Gibson SP, et al. Phase Ib Study of Immune Biomarker Modulation with Neoadjuvant Cetuximab and TLR8 Stimulation in Head and Neck Cancer to Overcome Suppressive Myeloid Signals. Clin Cancer Res. 2018; 24(1): 62-72.

4. Kansy BA, Concha-Benavente F, Srivastava RM, et al. PD-1 Status in CD8(+) T Cells Associates with Survival and Anti-PD-1 Therapeutic Outcomes in Head and Neck Cancer. Cancer Res. 2017; 77(22): 6353-6364.

5. Ben-Dayan MM, Ow TJ, Belbin TJ, et al. Nonpromoter methylation of the CDKN2A gene with active transcription is associated with improved locoregional control in laryngeal squamous cell carcinoma. Cancer Med. 2017; 6(2): 397-407.

6. Li YY, Chung GT, Lui VW, et al. Exome and genome sequencing of nasopharynx cancer identifies NF- $\kappa \mathrm{B}$ pathway activating mutations. Nat Commun. 2017; 8: 14121
7. Lesseur C, Diergaarde B, Olshan AF, et al. Genome-wide association analyses identify new susceptibility loci for oral cavity and pharyngeal cancer. Nat Genet. 2016; 48(12): 1544-1550.

8. Yuan Y, Sturgis EM, Zhu L, et al. A functional variant at the miRNA binding site in E2F1 gene is associated with risk and tumor HPV16 status of oropharynx squamous cell carcinoma. Mol Carcinog. 2017; 56(3): 1100-1106.

9. Economides MP, Amit M, Mahale PS, et al. Impact of chronic hepatitis C virus infection on the survival of patients with oropharyngeal cancer. Cancer. 2018; 124(5): 960-965.

10. Dahlstrom KR, Anderson KS, Cheng JN, et al. HPV Serum Antibodies as Predictors of Survival and Disease Progression in Patients with HPVPositive Squamous Cell Carcinoma of the Oropharynx. Clin Cancer Res. 2015; 21(12): 2861-9.

11. Gendler S. MUC1, the renaissance molecule J Mammary Gland Biol Neoplasia; 2001; 6(3): 339-53.

12. Kufe D W. Mucins in cancer: function, prognosis and therapy. Nat Rev Cancer 2009; 9(12): 874-885

13. Zhang L, Vlad A, Milcarek C, Finn OJ. Human mucin MUC1 RNA undergoes different types of alternative splicing resulting in multiple isoforms. Cancer Immunol Immunother. 2013; 62(3): 423-35.

14. Nath S, Mukherjee P. MUC1: a multifaceted oncoprotein with a key role in cancer progression. Trends in Molecular Medicine 2014; 20(6): 332-342.

15. Croce MV, Price MR, Segal-Eiras A. Detection and isolation of MUC1 mucin from larynx squamous cell carcinoma. Pathol Oncol Res. 2000; 6(2): 93-9.

16. Nakamori $S$, Kameyama M, Imaoka $S$, et al. Increased expression of sialyl Lewisx antigen correlates with poor survival in patients with colorectal carcinoma: clinicopathological and immunohistochemical study. Cancer Res. 1993; 53(15): 3632-7.

17. Nakamori $S$, Kameyama $M$, Imaoka $S$, et al. Involvement of carbohydrate antigen sialyl Lewis(x) in colorectal cancer metastasis. Dis Colon Rectum. 1997; 40(4): 420-31.

18. Croce MV, Colussi AG, Zambelli A, et al. Establishment and characterization of a cell line (T201) derived from a human larynx squamous cell carcinoma. Int J Oncol. 2001; 18(4): 729-35.

19. Croce MV, Rabassa ME, Price MR, et al. MUC1 mucin and carbohydrate associated antigens as tumor markers in head and neck squamous cell carcinoma. Pathol Oncol Res. 2001; 7(4): 284-91.

20. Croce MV, Rabassa ME, Pereyra A, et al. Influence of sialic acid removal on MUC1 antigenic reactivity in head and neck carcinoma. Pathol Oncol Res. 2005; 11(2): 74-81.

21. Rabassa ME, Croce MV, Pereyra A, et al. MUC1 expression and antiMUC1 serum immune response in head and neck squamous cell carcinoma (HNSCC): a multivariate analysis. BMC Cancer. 2006; 6: 253.

22. Croce MV, Isla-Larrain M, Rabassa ME, et al. Lewis $x$ is highly expressed in normal tissues: a comparative immunohistochemical study and literature revision. Pathol Oncol Res. 2007; 13(2): 130-8.

23. Mehla K, Singh PK. MUC1: a novel metabolic master regulator. Biochim Biophys Acta. 2014; 1845(2): 126-35.

24. Haddon L, Hugh J. MUC1-mediated motility in breast cancer: a review highlighting the role of the MUC1/ICAM-1/Src signaling triad. Clin Exp Metastasis. 2015; 32(4): 393-403.

25. Apostolopoulos V, Stojanovska L, Gargosky SE. MUC1 (CD227): a multi-tasked molecule. Cell Mol Life Sci. 2015; 72(23): 4475-500.

26. Hanson RL, Hollingsworth MA. Functional Consequences of Differential O-glycosylation of MUC1, MUC4, and MUC16 (Downstream Effects on Signaling). Biomolecules. 2016; 6(3). pii: E34. 
27. Rajabi H, Kufe D. MUC1-C Oncoprotein Integrates a Program of EMT, Epigenetic Reprogramming and Immune Evasion in Human Carcinomas. Biochim Biophys Acta. 2017; 1868(1): 117-122.

28. Blanas A, Sahasrabudhe NM, Rodríguez E, et al. Fucosylated Antigens in Cancer: An Alliance toward Tumor Progression, Metastasis, and Resistance to Chemotherapy. Front Oncol. 2018; 23(8): 39.
29. Croce MV, Rabassa ME, Pereyra A, et al. Differential expression of MUC1 and carbohydrate antigens in primary and secondary head and neck squamous cell carcinoma. Head Neck. 2008; 30(5): 647-57.

30. Rabassa ME, Pereyra A, Pereyra L, et al. Lewis x Antigen is Associated to Head and Neck Squamous Cell Carcinoma Survival. Pathol Oncol Res. 2017 Jul 5. 